\title{
O Índice de Desafios da Gestão Municipal - IDGM nas capitais da região Nordeste do Brasil no ano de 2018
}

The Municipal Management Challenge Index - IDGM in the capitals of the Northeast region of Brazil in 2018

EI Índice de Desafío de Gestión Municipal - IDGM en las capitales de la región Nordeste de Brasil en 2018

Michael Douglas Sousa Leite ORCID: https://orcid.org/0000-0002-9356-1872

Faculdade Católica Santa Teresinha, Brasi

E-mail: michaeldouglas_adm@hotmail.com

Roberlúcia Araújo Candeia ORCID: https://orcid.org/0000-0002-6783-8794 Universidade Federal de Campina Grande, Brasil E-mail: roberlucia.araujo@professor.ufcg.edu.br

Marcos Macri Olivera

ORCID: https://orcid.org/0000-0001-9446-4727

Universidade Federal de Campina Grande, Brasil E-mail: macri.ccjs@gmail.com

Verônica Cristian Soares de Belchior ORCID: https://orcid.org/0000-0003-2357-9599 Faculdades Integradas do Ceará, Brasil

E-mail: prof_veronicabelchior@hotmail.com

Thaise de Abreu Brasileiro Sarmento ORCID: https://orcid.org/0000-0003-0390-805X Universidade Federal de Campina Grande, Brasil E-mail: thaiseabreu@hotmail.com

Wendell de Almeida Lacerda ORCID: https://orcid.org/0000-0002-9737-1013 Faculdades Integradas do Ceará, Brasil E-mail:wendelllacerada@gmail.com

Wanessa Arnaud Moura ORCID: https://orcid.org/0000-0003-0274-5527 Universidade Federal de Campina Grande, Brasil E-mail: wanessa_arnaud@hotmail.com Tiago Douglas Cavalcante Carneiro ORCID: https://orcid.org/0000-0003-1183-1718

Faculdade Católica Santa Teresinha, Brasil E-mail: tiagocarneirocaico@gmail.com

Sandra Maijane Soares de Belchior

ORCID: https://orcid.org/0000-0001-5807-2259 Faculdades Integradas do Ceará, Brasil E-mail: sandrabelchior@hotmail.com Vinícius Campos de França ORCID: https://orcid.org/0000-0002-2512-8241 Universidade Federal de Campina Grande, Brasil E-mail: viniciuscamposf@gmail.com

Oziel Oliveira da Silva ORCID: https://orcid.org/0000-0002-8016-1220 Universidade Federal de Campina Grande, Brasil E-mail: ozisoliv@gmail.com

Moema da Nóbrega Euclides Lima ORCID: https://orcid.org/0000-0001-6774-0925 Universidade Federal de Campina Grande, Brasil E-mail: moemanelima@gmail.com 


\author{
Cleuton de Sousa Silva \\ ORCID: https://orcid.org/0000-0002-4254-1564 \\ Faculdades Integradas do Ceará, Brasil \\ E-mail: cs.arquitetura@hotmail.com \\ Alysson Emanuel de Sousa Nogueira \\ ORCID: https://orcid.org/0000-0003-2683-4794 \\ Faculdades Integradas de Patos, Brasil \\ E-mail: alyssonpsi78@gmail.com \\ Gisely Gabriela Bezerra de Sousa \\ ORCID: https://orcid.org/0000-0002-0010-0541 \\ Faculdades Integradas do Ceará, Brasil \\ E-mail: giselysousa@sousaadvocacia.com \\ Nijair Araújo Pinto \\ ORCID: https://orcid.org/0000-0003-0233-5501 \\ Faculdades Integradas do Ceará, Brasil \\ E-mail: nijair@hotmail.com
}

\begin{abstract}
Resumo
O percurso da história brasileira retrata a vivencia de problemas de cunho socioeconômico, capazes de comprometer a gestão e desempenho dos municípios, Estados e da União. Neste aspecto, os indicadores existem com a finalidade de avaliar o grau do comprometimento dos gestores, contribuindo efetivamente para demonstrar os resultados das ações desses junto as comunidades. O presente artigo tem como objetivo analisar o Índice de Desafios da Gestão Municipal e a alocação de recursos em algumas funções estratégicas governamentais nas capitais da região do Nordeste no ano de 2018. Para tanto a presente pesquisa corresponde a um estudo quantitativo, documental e exploratório, com uso da estatística descritiva, onde os dados foram obtidos através da base de dados da Finanças do Brasil (FINBRA) divulgados pela Secretaria do Tesouro Nacional (STN) e do site MACROPLAN. Os resultados mostram que dentre as cidades pesquisadas Fortaleza, João Pessoa, Teresina e Recife apresentam os melhores indicadores. Os piores indicadores foram de Maceió e Aracajú. Como sugestões de pesquisas futuras espera-se que possa continuar a investigação ao longo dos anos, bem como verificar as diferenças regionais, variáveis demográficas, representação política e econômica.
\end{abstract}

Palavras-chave: Gestão pública; Indicadores de desempenho; Desenvolvimento econômico e social.

\begin{abstract}
The trajectory of Brazilian history portrays the experience of socioeconomic problems, capable of compromising the management and performance of municipalities, States and the Union. In this respect, the indicators exist in order to assess the degree of commitment of managers, effectively contributing to demonstrate the results of their actions to the communities. This article aims to analyze the Municipal Management Challenges Index and the allocation of resources in some strategic government functions in the capitals of the Northeast region in 2018. For this purpose, this research corresponds to a quantitative, documentary and exploratory study, using descriptive statistics, where the data were obtained through the Brazilian Finance database (FINBRA) released by the National Treasury Secretariat (STN) and the MACROPLAN website. The results show that among the cities surveyed Fortaleza, João Pessoa, Teresina and Recife present the best indicators. The worst indicators were from Maceió and Aracajú. As suggestions for future research it is hoped that you can continue the investigation over the years, as well as verify regional differences, demographic variables, political and economic representation.
\end{abstract}

Keywords: Public management; Performance indicators; Economic and social development.

\title{
Resumen
}

La trayectoria de la historia brasileña retrata la experiencia de problemas socioeconómicos, capaces de comprometer la gestión y el desempeño de los municipios, los Estados y la Unión. En este sentido, los indicadores existen para evaluar el grado de compromiso de los gestores, contribuyendo efectivamente a demostrar el resultados de sus acciones a las comunidades. Este artículo tiene como objetivo analizar el Índice de Desafíos de la Gestión Municipal y la asignación de recursos en algunas funciones estratégicas de gobierno en las capitales de la región Nordeste en 2018. Para ello, esta investigación corresponde a un estudio cuantitativo, documental y exploratorio, utilizando estadística descriptiva, donde los datos se obtuvieron a través de la base de datos de Finanzas Brasileñas (FINBRA) difundida por la Secretaría del Tesoro Nacional (STN) y el sitio web MACROPLAN. Los resultados muestran que entre las ciudades encuestadas Fortaleza, João Pessoa, Teresina y Recife presentan los mejores indicadores. Los peores indicadores fueron los de Maceió y Aracajú. Como sugerencias para futuras investigaciones se espera que se pueda continuar la investigación a lo largo de los años, así como verificar diferencias regionales, variables demográficas, representación política y económica.

Palabras clave: Gestión pública; Indicadores de desempeño; Desarrollo económico y social. 


\section{Introdução}

A Constituição Federal de 1988, representa um marco imprescindível para a autonomia e também independência dos municípios. Apesar disso, o panorama da política brasileira aponta para uma concepção ainda tradicionalista, onde os municípios são entre os entes federados (Estados e União) os que tem a menor importância no cenário do poder e na agenda gerada em Brasília (Brasil, 1988; Forte, 2020).

O município é visualizado como o "locus" do cidadão, representando o núcleo das relações, ou seja, o local onde a vida é de fato concretizada. Portanto, o atual cenário de execução dos programas e das ações federais apresentam como principal fim o bem-estar do cidadão (Brasil, 2008; Leite et al., 2018).

Entretanto, o percurso da história brasileira retrata a vivencia de problemas de cunho socioeconômico como: a inflação, a recessão, a carência dos recursos para os investimentos e a ausência de instrumentos que sejam eficazes para o planejamento e para o controle dos gastos públicos. Concomitante, a história da gestão pública brasileira está marcada por uma série de ações de mal condução da gestão, como a má distribuição dos recursos, a falta de responsabilidade com a coisa pública, a utilização de recursos de maneira irresponsável e a falta de compromisso (Oliveira, Peter \& Meneses, 2010). Essas razões foram as que geraram os altos índices da corrupção e do endividamento.

Desta forma, é possível deduzir que as políticas públicas precisam começar a nível municipal e, devem estar envolvidas com as prioridades e as demandas locais pré-estabelecidas. Em tempos de crise econômica e recessão, os entes públicos enfrentam grandes desafios, o que demandou do setor uma série de mudanças e transformação ao longo dos anos, no intuito de democratizar e tornar transparente a gestão pública (Leite et al., 2018; Diniz, Pontes \& Pinheiro, 2020). Frente a este panorama o presente artigo tem como problemática compreender até que determinado ponto a gestão municipal pública fiscal estaria conexa com os indicadores de municipais na perspectiva local, com dados das Capitais do Nordeste.

A essência da gestão eficaz, segundo Carvalho (2011) objetiva implementar o bem-estar social dos indivíduos, sobremodo no que diz respeito à execução das políticas de atendimento de suas necessidades básicas, tais como: a educação, a saúde, a moradia e as medidas assistenciais primárias, promovendo o bem de todos e minimizando as desigualdades. Nesta perspectiva, o Estado precisa planejar as suas ações com a finalidade de atingir propósitos e resultados satisfatórios. Para isso, faz-se preciso envolver um ciclo integrado de gestão pública transparente, responsável, bem-planejada e compreensível aos administrados.

Vale destacar que com a crescente corrupção, naturalmente, há a perca da legitimidade e credibilidade por parte população, o que exige dos gestores públicos a necessidade de executar mudanças na forma de administrar e prestar contas dos recursos públicos (Leite et al., 2018; Diniz, Pontes \& Pinheiro, 2020). Desse modo, o objetivo deste artigo é analisar o Índice de Desafios da Gestão Municipal e a alocação de recursos em algumas funções estratégicas governamentais nas capitais da região do Nordeste no ano de 2018.

\section{Indicadores de Desempenho da Administração Pública}

Um indicador é definido enquanto um parâmetro/padrão que mensura a diferença entre as situações desejadas e atual situação, ou seja, ele indica quais são as dificuldades que precisam ser confrontadas (Zucatto et al., 2009; Leite et al., 2018). Assim, são ferramentas imprescindíveis para controle da gestão, não somente na administração privada, mas sobretudo na administração pública, por tornarem a gestão mais diáfanas e facilitarem o diálogo e comunicação entre os diversos grupos organizados. Pode-se descrever, que os indicadores na gestão são importantes ferramentas para a administração e, ainda, um instrumento para a fiscalização da gestão por parte dos movimentos populares (Barbalho \& Arão, 2015; Silva, 2020).

Colaborando, Rodrigues e Miranda (2015) relatam que os indicadores são formas de representação quantificáveis das características dos serviços e dos processos. São dispostos geralmente pela instituição para melhoramento e controle da qualidade 
e do desempenho dos processos. Os autores supramencionados destacam que os indicadores estão associados às características da qualidade dos serviços prestados, julgadas pelos próprios usuários (Réus \& Andion, 2018; Castro \& Carvalho, 2018).

Nessa perspectiva, Zucatto et al., (2009), relatam que o uso de indicadores está conexo à necessidade de tomar as decisões sobre os fatos, garantindo um processo de administração sobre variáveis internas e também externas da instituição, assim os indicadores de desempenho são essenciais para gerenciamento dos processos, pois aquilo que não se consegue mensurar, não se pode gerencia.

O uso dos indicadores pode ser otimizado ainda da seguinte forma, com a análise crítica e também o uso de atualizações; com a implementação das ações corretivas e de recomendações; com o acesso dos indicadores a todos os usuários que dele precisam; com a redução de tempo, desde a coleta até a análise crítica dos dados com as melhorias e por último com a relação dos indicadores (Zucatto et al., 2009; Castro \& Carvalho, 2018).

Para o Tribunal de Contas da União - TCU (2017), indicador de desempenho é uma fração, um número, porcentagem ou razão em que se mensura um aspecto, com o escopo de conferir este padrão com as metas preestabelecidas. Alguns autores (Brasil, 2009; Zucatto et al., 2009; Leite et al., 2018; Castro \& Carvalho, 2018) conceituam o indicador de desempenho como uma ferramenta de mensuração quantitativa/qualitativa de determinados aspectos do desempenho.

Entre as possibilidades diversas de informações que se pode ser produzida e escolhida para a avaliação dos programas de governos, se encontram os mecanismos de exame denominados de indicadores de desempenho que, segundo Filho \& Fialho (2015), são aproximações da realidade da gestão e trazem uma visão do resultado que se almeja mensurar. Os autores sustentam também que esses indicadores sempre devem receber dos seus clientes/usuários uma interpretação conexa com o contexto do qual estão inseridos.

Filho e Fialho (2015) acrescentam ainda, que os indicadores não servem somente para o seu processo de planejamento, controle, execução e monitoramento, mas outros stakeholders, como os organismos da Administração Pública (setoriais, centrais e etc.), os envolvidos em temáticas transversais, os administradores da agenda prioritária de governo, os outros Poderes e as instituições privadas (Bittencourt, 2006; Rodrigues \& Miranda, 2015; Réus \& Andion, 2018).

A utilização de indicadores se torna imperativa, pois se configura como um instrumento funcional/operacional para monitoramento da realidade com a finalidade de formulação e também reformulação das políticas (Bittencourt, 2006; Réus \& Andion, 2018). O Brasil é um país ainda marcado pela desigualdade, onde a cada dia emergem demandas novas sociais.

\section{3. Índice de Desafios da Gestão Municipal (IDGM)}

O Índice Desafios da Gestão Municipal - IDGM é um índice sinóptico que, por meio de uma categorização (ranking), compara a situação e variação da posição das 100 maiores cidades, que juntas representam mais da metade (50\%) do Produto Interno Bruto - PIB do país. O indicador abrange um período determinado e busca auxiliar os gestores a compreender e subsidiar os caminhos para enfrentar e também superar os desafios ao desenvolvimento, sobremodo no atual cenário de profundas restrições fiscais e crise econômica (Macroplan, 2020).

O IDGM abrange um total de 16 (dezesseis) indicadores em 4 (quatro) áreas definidas (saneamento, segurança, educação e saúde) e apresenta painéis que permitem uma ampla visão dos municípios nos campos: situação fiscal, transparência, gestão pública e economia, como mostra a (Figura 1) abaixo: 
Figura 1 - IDGM, indicadores e panorama dos municípios.

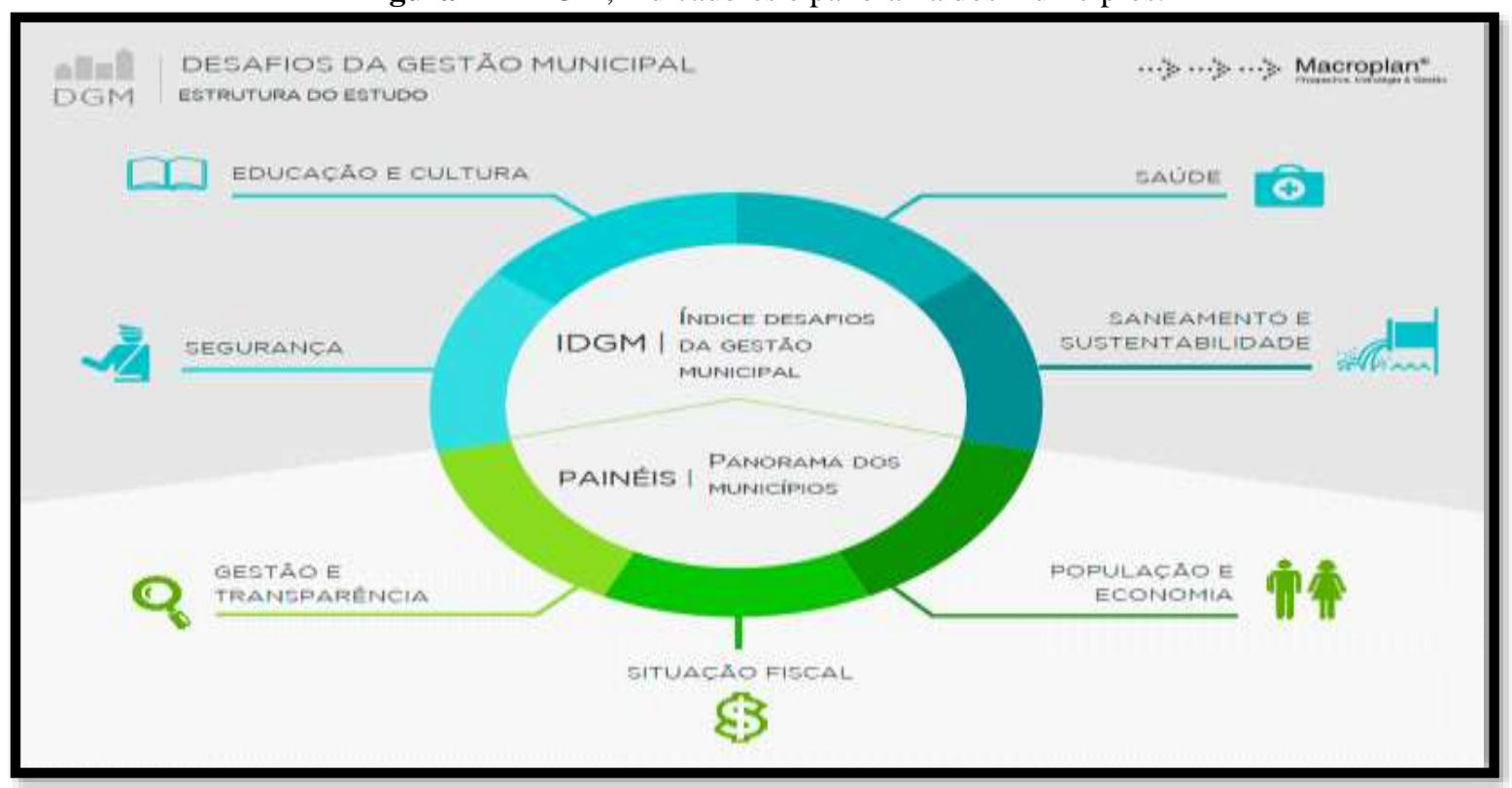

Fonte: Macroplan (2018).

A empresa Macroplan criou o IDGM com ferramenta semelhante à do Índice de Desenvolvimento Humano - IDH do Programa das Nações Unidas para o Desenvolvimento - PNUD, variando de (0 a 1), onde quanto mais perto de 1, melhor a performance do município. Desse modo, o estudo colabora uma análise temporal e comparativa das cidades, permitindo identificar os problemas principais e as soluções na área da gestão municipal (Macroplan, 2020).

A fórmula usada para calcular os indicadores de desempenho, cujo o crescimento significa melhoria direta, é a apresentada na sequência.

\section{$I i=V i-$ Valor mínimo \\ Valor maximo - Valor mínimo}

Onde, Ii é o índice do município e Vi é o valor do indicador no município i. Se o crescimento do indicador significa piora da situação do município, a fórmula é modificada e passa a ter a seguinte notação:

\section{$I i=V i-$ Valor máximo \\ Valor mínimo-Valor maximo}

Para os indicadores de cobertura e do IDEB, os valores mínimos e também máximos são os teóricos, podendo variar de $0 \%$ a $100 \%$, no caso do IDEB de 0 a 10. Os limites dos outros indicadores foram definidos a segundo os valores máximos e mínimos das variáveis nos 100 municípios, no período considerado, dependendo da disponibilidade dos dados de cada variável (Macroplan, 2020).

Os pesos de cada indicadores e das áreas que o compõem são definidos a partir de análise par-a-par dos indicadores para cada área e, em seguida, das próprias áreas, seguindo a metodologia da Análise Hierárquica de Prioridades - AHP (Macroplan, 2020). 


\section{Metodologia}

A presente pesquisa trata-se de um estudo quantitativo documental e exploratório, com uso da estatística descritiva. Para Yin (2015) a pesquisa quantitativa é uma classificação do método que utiliza técnicas diferentes estatísticas para quantificar informações para um determinado estudo. Em relação à pesquisa exploratória Gil (2019) destaca que ela tem o objetivo de proporcionar visão geral, de aproximar-se dos fatos e geralmente constitui como a primeira etapa de uma investigação mais ampla. Richardson (2012) afirma que a pesquisa exploratória é utilizada em questões ainda pouco exploradas e estudadas, fazendo assim familiarizar-se e esclarecer essas questões.

O estudo foi realizado com as capitais dos estados da região Nordeste do País no ano de 2018. Em comparação com outras regiões brasileiras, o Nordeste tem a segunda maior população, o terceiro maior território, o segundo maior colégio eleitoral, o menor IDH (2017) e o terceiro maior PIB (2019), o que torna uma região relevante para estudos correlatos à gestão governamental.

Os dados foram obtidos através da base de dados Finanças do Brasil (FINBRA) divulgados pela Secretaria do Tesouro Nacional (STN) e pelo site Macroplan. Assim, a coleta de dados foi feita, no mês de setembro/outubro de 2020.

Foram selecionados os indicadores de gestão pública, de maneira que fosse possível identificar sua relação com o desenvolvimento das 9 cidades, na Região do Nordeste. A priori foi feita uma análise dos indicadores financeiros e posteriormente a relação do IDGM com os demais indicadores.

Para tanto, dez indicadores (critérios) de gestão pública para mensurar o desenvolvimento econômico dos municípios envolvendo Saúde, Educação, Segurança, População e Economia e Saneamento, conforme mostra o Quadro 1.

Quadro 1 - Indicadores utilizados no estudo.

\begin{tabular}{|c|c|c|c|}
\hline Indicador & Fórmula & Objetivo & Relação \\
\hline FPM per capita & $\begin{array}{l}\text { FMP } \underset{\text { per }}{\text { capita }}= \\
\text { arrecadado/População total }\end{array}$ & $\begin{array}{l}\text { Avaliar a capacidade da captação } \\
\text { de transferências constitucionais } \\
\text { da União }\end{array}$ & Positiva. \\
\hline PIB per capita & $\begin{array}{l}\text { PIB per capita = PIB total/População } \\
\text { total }\end{array}$ & $\begin{array}{l}\text { é um indicador que ajuda a medir o } \\
\text { grau de desenvolvimento } \\
\text { económico de uma região }\end{array}$ & Positiva \\
\hline IDH-M & $\begin{array}{l}\text { O índice, considerado como indicador, } \\
\text { varia de Onenhum desenvolvimento } \\
\text { humano) a } 1 \text { (desenvolvimento } \\
\text { humano total). }\end{array}$ & $\begin{array}{l}\text { Esse indicador apresenta } \\
\text { relevância, pois fornece um retrato } \\
\text { do desenvolvimento por meio de } \\
\text { aspectos de educação, renda e } \\
\text { longevidade. }\end{array}$ & Positiva. \\
\hline Gastos de saúde per capita & $\begin{array}{l}\text { Gastos de saúde per capita }=\text { Gastos em } \\
\text { saúde/População total }\end{array}$ & $\begin{array}{l}\text { Avaliar os gastos per capita com a } \\
\text { saúde no município }\end{array}$ & Positiva. \\
\hline Gastos em educação per capita & $\begin{array}{l}\text { Gastos em educação per capita }=\text { Total } \\
\text { gastos em educação/População total }\end{array}$ & $\begin{array}{l}\text { Avaliar os gastos per capita com a } \\
\text { educação no município }\end{array}$ & Positiva. \\
\hline Gastos em segurança per capita & $\begin{array}{l}\text { Gastos em segurança per capita = Total } \\
\text { gasto em segurança / População total }\end{array}$ & $\begin{array}{l}\text { Avaliar os gastos per capita com } \\
\text { segurança no município }\end{array}$ & Positiva. \\
\hline $\begin{array}{l}\text { Gastos em saneamento per } \\
\text { capita }\end{array}$ & $\begin{array}{l}\text { Gasto em saneamento per capita = } \\
\text { Total gasto em cultura / População } \\
\text { Total }\end{array}$ & $\begin{array}{l}\text { Avaliar os gastos per capita com } \\
\text { saneamento no município }\end{array}$ & Positiva. \\
\hline $\begin{array}{l}\text { Índice Desafios da Gestão } \\
\text { Municipal (IDGM) }\end{array}$ & $\begin{array}{l}\text { Consolida } 16 \text { indicadores em } 4 \text { áreas de } \\
\text { resultado (educação, saúde, segurança } \\
\text { e saneamento) }\end{array}$ & $\begin{array}{l}\text { O estudo fornece uma análise } \\
\text { comparativa e temporal das } \\
\text { cidades brasileiras que permite } \\
\text { identificar os principais problemas } \\
\text { e as soluções no âmbito da gestão } \\
\text { municipal }\end{array}$ & Positiva \\
\hline
\end{tabular}

Fonte: Adaptado de Leite et al. (2018). 
A justificativa da seleção dos indicadores está pautada na sua importância para o estudo. Através desses indicadores é possível aferir o desempenho da gestão e identificar onde estão seus problemas, possibilitando o aperfeiçoamento das ações. Quando se utiliza indicadores de desempenho, aumenta-se a eficiência, auxilia na tomada de decisão, entre outros benefícios. Dessa forma, é importante para as organizações que desenvolvam bons indicadores e que transmitam aos colaboradores a importância destes (Leite et al., 2018).

Para Castro \& Carvalho (2017) e Leite et al., (2018), após a definição de qual variáveis é necessário escolher o tipo de influência que cada uma delas, possui com o entorno geral, isso significa dizer, cada variável mede uma situação e a mensuração dos índices possibilita a identificação das relações positivas/negativas para o seu desenvolvimento.

Os dados obtidos foram analisados através do Software Excel (2013), sendo utilizado o método comparativo que segundo Gil (2019) pode ser considerada com um processo de construção do conhecimento nas ciências sociais. Assim, pode-se ser descoberto regularidades, padrões e transformações, continuidades, diferenças e semelhanças sobre os fenômenos sociais.

\section{Resultados e Discussão}

A seguir são apresentados os resultados relacionados aos indicadores considerados no estudo. A análise foi feita de forma comparativa entre os nove municípios analisados.

A Tabela 2 demonstra as variáveis trabalhadas neste estudo para cada um dos municípios da amostra, ou seja, população, o Fundo de Participação do Município per capita (FPM), o Índice de desenvolvimento Humano (IDH), Produto Interno Bruto (PIB) per capita e o Índice Desafios da Gestão Municipal (IDGM).

Tabela 1 - População, FPM, IDH-M, PIB per capita e IDGM.

\begin{tabular}{lrcccc}
\hline \multicolumn{1}{c}{ Cidades } & População & $\begin{array}{c}\text { FPM } \\
\text { per capita }\end{array}$ & IDH-M & $\begin{array}{c}\text { PIB } \\
\text { per capita }\end{array}$ & IDGM \\
\hline São Luís & 1.101 .884 & $\mathrm{R} \$ 12.264$ & 0,768 & $\mathrm{R} \$ 26.154,25$ & 0,519 \\
Teresina & 864.845 & $\mathrm{R} \$ 12.890$ & 0,751 & $\mathrm{R} \$ 22.597,68$ & 0,555 \\
Fortaleza & 2.669 .342 & $\mathrm{R} \$ 15.438$ & 0,754 & $\mathrm{R} \$ 23.045,09$ & 0,584 \\
Natal & 884.122 & $\mathrm{R} \$ 17.169$ & 0,763 & $\mathrm{R} \$ 24.890,01$ & 0,525 \\
João Pessoa & 809.015 & $\mathrm{R} \$ 14.774$ & 0,763 & $\mathrm{R} \$ 23.345,93$ & 0,575 \\
Recife & 1.645 .727 & $\mathrm{R} \$ 17.777$ & 0,768 & $\mathrm{R} \$ 30.477,73$ & 0,546 \\
Maceió & 1.018 .948 & $\mathrm{R} \$ 14.724$ & 0,721 & $\mathrm{R} \$ 20.853,41$ & 0,500 \\
Aracaju & 657.013 & $\mathrm{R} \$ 17.154$ & 0,771 & $\mathrm{R} \$ 25.717,68$ & 0,526 \\
Salvador & 2.872 .347 & $\mathrm{R} \$ 16.931$ & 0,759 & $\mathrm{R} \$ 20.796,62$ & 0,553 \\
& & & & & \\
\hline
\end{tabular}

Fonte: Dados da Pesquisa (2020).

O município mais populoso é a cidade de Salvador com 2.872.347 de habitantes, seguido por Fortaleza com 2.669.342, Recife 1.645.727 e São Luís 1.101.884. O município que apresentou maior FPM per capita foi Recife (R\$17.777), seguido por Natal ( $R$ \$ 17.169) e Aracaju ( $R$ \$ 17.154). No PIB per capita Recife apresentou o maior rendimento per capito com $R \$ 30.477,73$ e Salvador o pior entre as capitais do nordeste com $\mathrm{R} \$ 20.796,62$.

Para Duarte e Lima (2013), a qualidade da gestão em relação a área fiscal é determinada de proporcional maneira ao desenvolvimento do município, ou seja, quanto melhor comporta-se a gestão, são maiores as chances de os municípios socialmente desenvolver-se. Isso mostra que os serviços estão sendo disponibilizados para a sociedade e que as cidades mostram uma dimensão financeira boa e, assim, existe uma maior possibilidade de aumentar os indicadores de qualidade e de desenvolvimento, podendo uma boa gestão influencia no desenvolvimento municipal. 
No Índice de Desenvolvimento Humano a melhor capital do Nordeste foi Aracajú $(0,771)$, seguido bem de perto por São Luís e Recife ambos com (0,768). A pior das cidades no IDH foi Maceió com (0,721). Segundo Marino et al., (2016), o IDH é um índice universal, usado para se mensurar o desenvolvimento humano de uma região ou mesmo países, tendo por finalidade representar a complexidade em termos do desenvolvimento humano que ele proporciona. Para isso, são analisados três elementos que são essenciais para a vida dos indivíduos: renda, longevidade e educação, sendo que esses elementos são caracterizados por um grupo de indicadores.

Neste aspecto, Hungaro (2016) relata uma relação entre variáveis econômicas (os investimentos realizados) e características socioeconômicas, demostrando que quanto maior o investimento o desenvolvimento municipal (IDH-M).

No Índice Geral dos Desafios da Gestão Municipal (IDGM) Fortaleza ficou em primeiro lugar $(0,584)$ dentre as capitais do Nordeste. Já no ranking de todas as cidades brasileiras Fortaleza ocupa a $56^{\circ}$ posição. No segundo lugar vem João Pessoa $(0,575)$ e dentre as capitais pior resultado ficou com Maceió com $(0,500)$.

Na Tabela 2 estão os gastos per capita da área da saúde e o IDGM Saúde de cada município.

Tabela 2 - Desempenho da Saúde Pública Municipal.

\begin{tabular}{lrrrrrr}
\hline \multirow{2}{*}{ Variável } & \multicolumn{2}{c}{ Gasto per capita } & \multicolumn{2}{c}{ IDGM Saúde } & \multicolumn{2}{c}{ Análise Comparativa } \\
& Valor & \multicolumn{1}{c}{ Score } & \multicolumn{1}{c}{ Valor } & \multicolumn{1}{c}{ Score } & \multicolumn{1}{c}{ Valor } & Classificação \\
\hline São Luís & 304,25 & 1,94 & 0,5160 & 0,84 & 0,434 & $2^{\circ}$ Lugar \\
Teresina & $2.557,11$ & 16,32 & 0,5600 & 0,92 & 0,056 & $9^{\circ}$ Lugar \\
Fortaleza & 598,23 & 3,82 & 0,6120 & 1,00 & 0,262 & $4^{\circ}$ Lugar \\
Natal & 156,73 & 1,00 & 0,5700 & 0,93 & 0,931 & $1^{\circ}$ Lugar \\
J. Pessoa & 673,97 & 4,30 & 0,5780 & 0,94 & 0,220 & $6^{\circ}$ Lugar \\
Recife & 473,96 & 3,02 & 0,5420 & 0,89 & 0,293 & $3^{\circ}$ Lugar \\
Maceió & $1.212,28$ & 7,73 & 0,5020 & 0,82 & 0,106 & $8^{\circ}$ Lugar \\
Aracaju & 859,79 & 5,49 & 0,5890 & 0,96 & 0,175 & $7^{\circ}$ Lugar \\
Salvador & 545,03 & 3,48 & 0,5440 & 0,89 & 0,256 & $5^{\circ}$ Lugar \\
\hline
\end{tabular}

Fonte: Dados da Pesquisa (2020).

Para o cálculo do Score se pautou na análise da relação positiva do IDGM (quanto maior melhor) e negativa para os gastos per capita (quanto menor melhor) entre cada um dos indicadores selecionados. Essa estratégia proporciona o ajuste dos valores observados nas variáveis, cujo valor mínimo é zero e valor máximo é igual um.

No primeiro lugar ficou com Natal, gastando apenas 156,73 reais por habitante, tem media 0,570 no IDGM saúde e na análise comparativa foi bem superior às outras capitais Nordestina. De acordo com o IDGM (2018), Fortaleza possui o melhor sistema de saúde dentre as capitais do Nordeste, ocupando a $34^{\circ}$ colocação entra as cidades brasileiras, posterior vem Aracajú $44^{\circ}$ colocação e João Pessoa $48^{\circ}$ colocação.

Na área da Saúde, Teresina foi o município que mais gastou em saúde, foram investidos no ano de $2018 \mathrm{R} \$ 2.557,11$ por habitante, em segundo lugar foi Maceió com R\$1.212,28. O relatório mostra que apesar de Maceió (87º colocada IDGM saúde) nos últimos anos vem investindo pesado na área da Saúde, subindo 13 cidades de 2006 a 2018, ela e Teresina são as cidades com a pior saúde pública dentre as capitais do Nordeste.

É unânime entre os peritos da área da saúde que um dos problemas principais a afetar o sistema público de Saúde no Brasil é a gestão ineficiente (Fenili, 2015). Uma gestão ineficiente em tempos de recursos escassos tem impacto direto na vida da população. Assim é preciso tornar o processo de gestão pública mais dinâmico, fluído e transparente, fazendo assim com que os recursos sejam bem aproveitados. É necessário identificar os gargalos, buscando apoio tecnológico, motivando a interação das equipes e adotando sistemas informatizados, transformando o processo de gestão entre outros passos (Saldiva \& Veras, 
2018). Vale ressaltar que o cenário de crise é capaz de inspirar projetos novos, muitos deles vindo depois a se tornar bemsucedidos (MV, 2017).

Corroborando com isto, Saldiva e Veras (2018) ressaltam que os recursos financeiros destinados à saúde no Brasil, embora não sejam ideais, não são diferentes de países com melhor assistência à população. Atualmente, o financiamento à saúde vem oscilando ao redor de $8 \%$ do PIB nos últimos anos. Comparando com países que oferecem o acesso universal, utilizam recursos pouco superiores aos do Brasil, como: Reino Unido (9,9\% do PIB) e Canadá (10,4\% do PIB). Uma simples leitura indica que haja ineficiência dos gastos públicos e não um dificuldades de subfinanciamento no caso brasileiro (Saldiva \& Veras, 2018).

Abaixo é destacado os dados na área da educação de cada município:

Tabela 3 - Desempenho da Educação Pública Municipal.

\begin{tabular}{lrccccc}
\hline & \multicolumn{2}{c}{ Gasto per capita } & \multicolumn{2}{c}{ IDGM Educação } & \multicolumn{2}{c}{ Análise Comparativa } \\
\multicolumn{1}{c}{ Variável } & Valor & Score & Valor & Score & Valor & Classificação \\
\hline São Luís & 206,45 & 2,18 & 0,4880 & 0,89 & 0,407 & $2^{\circ}$ Lugar \\
Teresina & $1.147,79$ & 12,13 & 0,5490 & 1,00 & 0,082 & $8^{\circ}$ Lugar \\
Fortaleza & 610,46 & 6,45 & 0,4960 & 0,90 & 0,140 & $4^{\circ}$ Lugar \\
Natal & 94,61 & 1,00 & 0,4340 & 0,79 & 0,791 & $1^{\circ}$ Lugar \\
J. Pessoa & 406,86 & 4,30 & 0,4050 & 0,74 & 0,172 & $7^{\circ}$ Lugar \\
Recife & 233,79 & 2,47 & 0,4460 & 0,81 & 0,329 & $3^{\circ}$ Lugar \\
Maceió & $1.266,90$ & 13,39 & 0,3450 & 0,63 & 0,047 & $9^{\circ}$ Lugar \\
Aracaju & 457,68 & 4,84 & 0,3710 & 0,68 & 0,140 & $6^{\circ}$ Lugar \\
Salvador & 470,38 & 4,97 & 0,3820 & 0,70 & 0,140 & $5^{\circ}$ Lugar \\
\hline
\end{tabular}

Fonte: Dados da Pesquisa (2020).

$\mathrm{Na}$ área da Educação o destaque novamente fica para Natal gastando 94,61 reais por habitante, em segundo lugar ficou São Luís. Apesar de Teresina, estar em $8^{\circ}$ lugar na análise comparativa, sendo o segundo em gastos com $\mathrm{R} \$ 1.147,79$, a cidade possui bons resultados na área. Segundo IDGM (2018), Teresina ocupa $38^{\circ}$ colocação apresentando o melhor índice das cidades pesquisas. Maceió apresentou o pior índice de educação, tanto nos gastos per capita, no IDGM, ocupando a $100^{\circ}$ colocação e na análise comparativa. Dados do Ministério da Educação e Cultura - MEC (2018) apontam como uma das cidades com o maior porcentual de analfabetismo do País, com 20,7\% (MEC, 2018).

Para Callegari (2018) o problema maior está em se gastar melhor, com eficácia e em ações que façam a diferença realmente. A autora menciona que estratégias como o estimulo ao ensino em tempo integral nas escolas e o investimento em formação de professores têm maior impacto, do que compra de computadores.

Segundo o relatório pela Secretaria do Tesouro Nacional - STN produzido, denominado "Aspectos fiscais na educação no Brasil", a contradição de um país que aparece entre os que mais se gastam com a educação, mas, aparece nas últimas colocações de programas de avaliações internacionais como o Pisa. O estudo mostra que o montante atual de gastos é suficiente, ou seja, o valor aplicado em educação não apresenta significativa correlação com o desempenho no ensino.

Apesar da forte pressão da sociedade para a aumente os gastos na área de educação, evidências mostram de que a baixa qualidade não se deve à falta de recursos. Essa observação não é específica ao Brasil, visto que já é estabelecida na literatura cientifica de que políticas baseadas na ampliação apenas de insumos educacionais são, de modo geral, ineficazes.

A Tabela 4, por sua vez, destaca os dados na área de segurança pública dos municípios. 
Tabela 4 - Desempenho da Segurança Pública Municipal.

\begin{tabular}{lrccccc}
\hline & \multicolumn{2}{c}{ Gasto per capita } & \multicolumn{2}{c}{ IDGM Segurança } & \multicolumn{2}{c}{ Análise Comparativa } \\
\multicolumn{1}{c}{ Variável } & Valor & Score & Valor & Score & Valor & Classificação \\
\hline São Luís & 0,20 & 1,00 & 0,5490 & 0,84 & 0,8369 & $1^{\circ}$ Lugar \\
Teresina & 5,55 & 27,75 & 0,5670 & 0,86 & 0,0311 & $3^{\circ}$ Lugar \\
Fortaleza & 7,38 & 36,90 & 0,6150 & 0,94 & 0,0254 & $5^{\circ}$ Lugar \\
Natal & 5,99 & 29,94 & 0,5260 & 0,80 & 0,0268 & $6^{\circ}$ Lugar \\
J. Pessoa & 25,75 & 128,75 & 0,6020 & 0,92 & 0,0071 & $4^{\circ}$ Lugar \\
Recife & 3,87 & 19,35 & 0,6560 & 1,00 & 0,0517 & $2^{\circ}$ Lugar \\
Maceió & 776,20 & $3.881,00$ & 0,5450 & 0,83 & 0,0002 & $9^{\circ}$ Lugar \\
Aracaju & 124,66 & 623,30 & 0,4370 & 0,67 & 0,0011 & $7^{\circ}$ Lugar \\
Salvador & 256,85 & $1.284,25$ & 0,5650 & 0,86 & 0,0007 & $8^{\circ}$ Lugar \\
\hline
\end{tabular}

Fonte: Dados da Pesquisa (2020).

Na área da segurança pública São Luís o obteve a melhor média na análise comparativa, gastando apenas 0,20 centavos por habitante possui índices bons na área de segurança.

Maceió foi a quem mais investiu $\mathrm{R} \$ 776,20$, e está entra as capitais mais violentas. A pior das capitais do nordeste em segurança foi Aracajú. Segundo levantamento realizado pelo Instituto de Pesquisa Econômica e Aplicada - IPEA (2018), Aracaju atualmente passou a ser a segunda capital mais violenta do Brasil. Pelos dados divulgados do Atlas da Violência (2018), Aracaju apresenta o índice de 73 homicídios por 100 mil habitantes.

No ranking do IDGM (2018), Recife apresentou a melhor segurança pública dentre as cidades pesquisadas ocupando $57^{\circ}$ lugar, posteriormente Fortaleza $68^{\circ}$.

A Tabela 5 apresenta os resultados na área do saneamento básico.

Tabela 5 - Desempenho do Saneamento Público Municipal.

\begin{tabular}{|c|c|c|c|c|c|c|}
\hline \multirow[b]{2}{*}{ Variável } & \multicolumn{2}{|c|}{ Gasto per capita } & \multicolumn{2}{|c|}{ IDGM Saneamento } & \multicolumn{2}{|c|}{ Análise Comparativa } \\
\hline & Valor & Score & Valor & Score & Valor & Classificação \\
\hline São Luís & 8,08 & 3,58 & 0,5630 & 0,66 & 0,184 & $3^{\circ}$ Lugar \\
\hline Teresina & 2,26 & 1,00 & 0,5530 & 0,64 & 0,645 & $1^{\circ}$ Lugar \\
\hline Fortaleza & 14,94 & 6,61 & 0,6730 & 0,78 & 0,119 & $4^{\circ}$ Lugar \\
\hline Natal & 5,75 & 2,55 & 0,6060 & 0,71 & 0,277 & $2^{\circ}$ Lugar \\
\hline J. Pessoa & 24,74 & 10,95 & 0,8470 & 0,99 & 0,090 & $5^{\circ}$ Lugar \\
\hline Recife & 96,15 & 42,54 & 0,6780 & 0,79 & 0,019 & $6^{\circ}$ Lugar \\
\hline Maceió & 358,01 & 158,41 & 0,7440 & 0,87 & 0,005 & $9^{\circ}$ Lugar \\
\hline Aracaju & 343,38 & 151,94 & 0,7220 & 0,84 & 0,006 & $8^{\circ}$ Lugar \\
\hline Salvador & 166,77 & 73,79 & 0,8580 & 1,00 & 0,014 & $7^{\circ}$ Lugar \\
\hline
\end{tabular}

Fonte: Dados da Pesquisa (2020).

No Saneamento Básico Teresina obteve a melhor média na análise comparativa, gastando 2,26 reais por habitante, em $2^{\circ}$ lugar foi Natal e $3^{\circ}$ São Luís. O resultado contrário com ranking do IDGM (2018) na área do saneamento básico, onde Salvador apresenta a melhor colocação dentre as cidades pesquisadas $27^{\circ}$ lugar, seguida por João Pessoa $30^{\circ}$ lugar. Dentre os piores resultados Teresina ocupa a $89^{\circ}$ posição, posteriormente São Luís $86^{\circ}$ e Natal $79^{\circ}$.

De acordo com Leoneti, Prado e Oliveira (2011) as políticas públicas direcionadas para a melhoria das condições de saneamento das comunidades são eficazes para diminuir a mortalidade infantil e pós-neonatal, período este em que os óbitos 
ocorrem devido a doenças relacionadas principalmente às condições do ambiente em que se habita. Tal fato confirma estudos que o aumento da cobertura populacional por sistemas de esgotamento pode contribuir diretamente para reduzir mais a mortalidade infantil no Brasil.

\section{Considerações Finais}

O presente trabalho teve como objetivo analisar os Indicadores de Desempenho e o Índice de Desafios da Gestão Municipal nas capitais da região do Nordeste no ano de 2018. Assim, a pesquisa apontou que das 9 cidades, Fortaleza tem a melhor saúde pública, Teresina a melhor educação, Salvador e João Pessoa o melhor Saneamento e Recife os melhores índices em Segurança Pública, essas 5 cidades estão com bons indicadores em todas as áreas pesquisadas com base no IDGM.

Todavia, combinando tais indicadores com os investimentos per capita, o destaque fica para Natal na análise comparativa, pois apresentou bons indicadores na área de educação e saúde, sendo a cidade que menos gastou entre as pesquisadas. Tal fato se deve, a uma gestão eficiente, com alocação de recursos eficazes, fazendo a diferença na vida da população.

$\mathrm{O}$ restante das cidades apresentou indicadores financeiros e sociais em situação delicada com índices muito baixos. Dentre as cidades, Maceió foi destaque negativo, pois foi a que mais gastou dinheiro do contribuinte e apresentou os piores resultados em educação e saúde. Aracajú apresentou péssimos resultados na Segurança Pública, sendo a $2^{\circ}$ cidade mais violenta do país. Maceió e Aracajú apresentaram os piores resultados no Saneamento Básico

Desta forma, fica claro a fragilidade da gestão municipal dos municípios brasileiros, que ainda na maioria dos casos utilizam uma gestão aos moldes antigos, trabalhando em prol de interesses próprios, privilegiando o imediatismo e ações não planejadas, desperdiçando recursos e prestando serviços insuficientes para atender as demandas sociais, além de caracterizadas pela baixa qualidade.

Como sugestões a estudos futuros espera-se que possa continuar a investigação ao longo dos anos, bem como verificar as diferenças regionais, variáveis demográficas, representação política e econômica, se estas mesmas influenciariam na gestão municipal bem como o desenvolvimento.

\section{Referências}

Barbalho, A., \& Arão, M. R. (2015). Orçamento participativo e Gestão Municipal: A experiência de Fortaleza (2005-2008). Ciências Sociais em Perspectiva, 27(14), 268-287.

Bittencourt, F. M. R. (2006). Indicadores de desempenho como instrumentos de gestão, auditoria e análise econômica. Revista de Conjuntura: Editada pelo Conselho Regional de Economia do Distrito Federal, Brasília, 23(6), 49-60.

Brasil. (1988). Constituição da República Federativa do Brasil. Senado.

Brasil. (2008). Responsabilidade na gestão pública: os desafios dos municípios. Câmara dos Deputados, Edições Câmara.

Callegari, V. G. (2018). Muito, pouco, mal, de forma irregular? Especialistas avaliam o gasto do Brasil com educação. Revista Gaúcha, 1(12), 1-10.

Carvalho, J. R. de C., Carvalho, E. K. M. de A., Holanda, F. M. de A., Curi, W. F., \& Curi, R. C. (2011). Metodologia para Avaliar a Sustentabilidade Ambiental de Municípios Utilizando Análise Multicritério. REUNIR - Revista de Administração, Contabilidade e Sustentabilidade, 1(1), 18-34.

Castro, S. H. R., \& Carvalho, M. G. de. (2017). Indicador de Efetividade da Gestão Municipal: Contribuição dos Tribunais de Contas para a melhoria da Gestão Pública. Sistemas, Cibernética e Informática, 1(14), 46-56.

Diniz, G. M., Pontes, P. A., \& Pinheiro, B. G. (2020). A relação entre a transparência digital dos municípios cearenses e seus indicadores políticos, sociais e econômicos. Rev. Controle (2)18, 133-163.

Filho, G. A. L., \& Fialho, T. M. M. (2015). Relação entre indicadores de gestão pública e de desenvolvimento dos municípios brasileiros. Cadernos Gestão Pública e Cidadania, (67)20, 277-295.

Fenili, R. R. (2015). Gestão de Materiais. ENAP. 
Research, Society and Development, v. 10, n. 3, e58110313735, 2021

(CC BY 4.0) | ISSN 2525-3409 | DOI: http://dx.doi.org/10.33448/rsd-v10i3.13735

Forte, E. C. (2020). Transparência pública e acesso à informação: uma análise do Portal da Transparência do município de Fortaleza. Rev. Controle, Fortaleza, (2) $18,429-456$

Gil, A. C. (2019). Métodos e Técnicas de Pesquisa Social. (7a ed.), Atlas.

Hungaro, L. A. (2016). Parceria público-privada municipal e a concretização de funções sociais da cidade: Habitação, saneamento básico e mobilidade urbana. Dissertação Mestrado, Universidade Federal do Paraná, Curitiba, PR, Brasil.

Instituto de Pesquisa Econômica e Aplicada (IPEA). (2018). Atlas da Violência: IPEA.

Leite, M. D. S., Olivera, M. M., Assis, L. F., Macri, L. M. S. R., \& Lima, R. A. de A. (2018). Comportamento de indicadores de gestão pública dos municípios de uma microrregião do interior da Paraíba. Revista Semana Acadêmica, 129(01), 1-19.

Leoneti, A, B., Prado, E. L., \& Oliveira, S. V. W. B de. (2011). Saneamento básico no Brasil: Considerações sobre investimentos e sustentabilidade para o século XXI. Rap: 2(45), 331-348.

Macroplan. (2018). Desempenho das 100 maiores cidades do Brasil: IDGM.

Marino, P. de B. L. P., Soares, R. A. S., De Luca, M. M. M., \& Vasconcelos, A. C. de. (2016). Indicadores de governança mundial e sua relação com os indicadores socioeconômicos dos países do Brics. Revista de Administração Pública. 5(50), 721-744.

Ministério da Educação e Cultura (MEC). (2018). Analfabetismo no país nos últimos oito anos. PNAD.

MV. Como prover uma Saúde Pública de qualidade em tempos de crise? Recuperado de https://mv.com.br/pt/blog/como-prover-uma-saude-publica-dequalidade-em-tempos-de-criser

Oliveira, F. H., Peter, M. G. A., \& Meneses, A. F. (2009). Lei de responsabilidade fiscal: Implicações nos indicadores sociais municipais. In: Congresso USP de Controladoria e Contabilidade, Anais, 1-14

Réus, I., \& Andion, C. (2018). Gestão Municipal e Desenvolvimento Sustentável Panorama dos Indicadores de Sustentabilidade nos Municípios Catarinenses. Revista Desenvolvimento em Questão, 45(16), 97-117.

Richardson, J. (2012). A pesquisa qualitativa crítica e válida. (3a ed.), Revista e Ampliada. Atlas.

Rodrigues, R. R., \& Miranda, A. R. A. (2015). Os Entraves ao Gerencialismo na Gestão Pública de um Município do Interior do Estado de São Paulo. Caderno de Estudos Interdisciplinares, Edição Especial Gestão Pública e Sociedade, 70-80.

Saldiva, P. H. N., \& Veras, M. (2018). Gastos públicos com saúde: breve histórico, situação atual e perspectivas futuras. Revista Estudos Avançados, 92(32), $47-61$.

Secretaria do Tesouro Nacional (STN). (2018). Boletim Resultado do Tesouro Nacional (RTN), 2018. https://www.gov.br/tesouronacional/pt-br

Silva, G. de O. da. (2020). O Índice de Efetividade da Gestão Municipal com indutor do aperfeiçoamento da gestão pública nos municípios paulistas. Cadernos, $5(1), 65-81$.

Tribunal de Contas da União (TCU). (2017). Relatório de Políticas e Programas de Governo 2017. TC 018.218/2017-2.

Zucatto, L. C., Sartor, U. M., Beber, S., \& Weber, R. (2009). Proposição de indicadores de desempenho na gestão pública. ConTexto, 16(9), 1-24.

Yin, R. K. (2015). O Estudo de caso. Bookman. 